\title{
The impact of current health-related quality of life on future health outlook in patients with eosinophilic granulomatosis with polyangiitis (Churg-Strauss syndrome)
}

\author{
Barbara Sokolowska • Wojciech Szczeklik • Olga Pilat • \\ Michał Wodkowski • Justyna Pieczątka • \\ Jolanta Gąsior • Katarzyna Szczeklik • \\ Anna Włudarczyk • Karolina Pacult • Jacek Musial
}

Received: 26 October 2012 / Revised: 17 December 2012 / Accepted: 3 January 2013 / Published online: 18 January 2013

(C) The Author(s) 2013. This article is published with open access at Springerlink.com

\begin{abstract}
Eosinophilic granulomatosis with polyangiitis (EGPA) is a rare, autoimmune small and medium vessel vasculitis. EGPA is accompanied by asthma and involves mainly the blood vessels of the lungs, gastrointestinal system, and peripheral nerves; however, the skin, kidneys, and heart may be also affected. To investigate if patients with EGPA experience reduced health-related quality of life (HRQOL), and the effect of this parameter on their own perception of future health outlook. Twenty-six EGPA patients are in disease remission and completed a customdesigned questionnaire and the Medical Outcomes Study Short Form 36 (SF-36). Using the RAND method, eight HRQOL dimensions were calculated: general health, physical functioning, emotional role limitations, physical role limitations, social functioning, mental health, bodily pain, and vitality. Using norm-based scores, the HRQOL of patients was compared with that of the general population. EGPA patients had decreased HRQOL across all eight dimensions of the SF-36. Patients with higher mental component score felt more positive about their future health, while patients with low physical component score were likely not to feel negatively about their future health. Also, $36 \%$ of older patients ( $>50$ years) had a positive outlook
\end{abstract}

B. Sokołowska $\cdot$ W. Szczeklik $\cdot$ O. Piłat $\cdot$ M. Wodkowski $\cdot$

J. Pieczątka J. Gąsior · K. Szczeklik • A. Włudarczyk •

K. Pacult $\cdot$ J. Musiał

Jagiellonian University Medical College, Krakow, Poland

B. Sokołowska $(\triangle)$

Department of Medicine, Jagiellonian University Medical College,

ul. Skawińska 8,

31-066 Krakow, Poland

e-mail: basiasok1@gmail.com compared to $47 \%$ of younger patients $(<50$ years $)$ and patients with a longer disease course were much less likely to have a positive outlook ( $30 \%$ positive) than those with a shorter course $(50 \%$ positive). Although not statistically significant, these correlations warrant further investigation with a larger patient population. Despite being in disease remission, EGPA patients had decreased quality of life, which in turn influenced their perception of their future health outlook.

Keywords Eosinophilic granulomatosis with polyangiitis . Quality of life · Vasculitis

\section{Introduction}

Eosinophilic granulomatosis with polyangiitis (EGPA), previously known as Churg-Strauss syndrome, is a noninheritable and nontransmissible disease affecting medium and small vessels. It is an autoimmune vasculitis leading to tissue necrosis with eosinophilia and eosinophil tissue infiltration [1]. EGPA is accompanied by asthma and involves mainly the blood vessels of the lungs, gastrointestinal system, and peripheral nerves; however, other organs such as skin, kidneys, and most notably the heart may also be affected $[2,3]$. Over the years, the prognosis for EGPA patients has improved tremendously. After the introduction of corticosteroids into therapy, 5-year mortality rates have decreased to $13.9 \%$ from previously reported 3 -month rates of approximately $50 \%[4,5]$. It can be safely said that, over the last decade, EGPA has been reduced to a chronic relapsing disease usually requiring indefinite medication and periodic follow-up. The question thus arises whether this 
improvement has had a proportionally positive impact on the quality of life in these patients. Additionally, the understanding of the term "health" has also changed dramatically during this time and - unsurprisingly - patients' expectations have evolved correspondingly. In fact, many of them expect not only to be physically and emotionally healthy but to also enjoy a quality of life similar to that of people unaffected by disease.

Quality of life is defined as an individual's perception of their position in life in the context of the culture and value systems in which they live and in relation to their goals, expectations, standards, and concerns [6]. Measurement of such a relative parameter is challenging. Standardized methods such as the health-related quality of life (HRQOL) Short Form 36 (SF-36) have become a useful tool in measuring the quality of life of distinct patient populations and comparing it to healthy populations at one moment in time. It is also used to monitor the impact of novel treatments methods on the quality of life over a prolonged period of time [7]. The additional advantage of using such tools is that they provide a uniform platform which facilitates the comparison of quality of life measurements across similar types of diseases [8].

Although SF-36 has been used in patients with many auto-immune diseases such as granulomatosis with polyangitiis disease (GPA, Wegener's granulomatosis) [9], systemic lupus erythematosus [10], newly diagnosed antineutrophil cytoplasmic antibody (ANCA)-associated vasculitis [8], there has been no study using this method that focuses solely on patients with eosinophilic granulomatosis with polyangiitis. EGPA patients suffer from a unique set of symptoms that affect their daily lives in ways different from other vasculitic diseases. This is partially due to the considerable burden that asthma places on patient's daily living activities, especially before a diagnosis is made [11]. Another question that has not been addressed is how patients' feel about their perceived future health outlook, an important yet rarely considered outcome measure [12], and what factors influence this perception.

These reasons prompted the investigation of whether current quality of life in EGPA patients exerts any influence on their future health outlook. Several other significant factors such as gender, employment, and duration of disease were also screened for correlation with future health outlook.

\section{Patients and methods}

Medical records from the Outpatient Allergy and Immunology Clinic of the Jagiellonian University Hospital in Cracow, Poland were searched to identify patients with EGPA. The diagnosis was verified before enrollment in all patients included in this study. As no diagnostic criteria are available for
EGPA, the American College of Rheumatology (ACR) classification criteria were used [13]. They are the most widely accepted criteria in this condition. They distinguish six key features of the disease, among them: asthma, peripheral blood eosinophilia of $>10 \%$, mono- or polyneuropathy, paranasal sinus abnormality, eosinophilic accumulation in tissues confirmed by a biopsy, and migratory infiltrates in lungs. At least four of the criteria listed above had to be confirmed in order to validate the diagnosis. A custom questionnaire regarding current symptoms, current medication regimen, and employment history, Short Form 36 Health Survey (QualityMetrics v. 2) as well as future health outlook were included in the survey package. The Short Form 36 Health Survey, a standardized questionnaire, is used to compile two key parameters: a mental component score (MCS) and a physical component score (PCS). An absolute score (0-100) was obtained for each parameter. Using the RAND method, a total of eight HRQOL dimensions were measured: general health $(\mathrm{GH})$, physical functioning (PF), emotional role limitations (RE), physical role limitations (RP), social functioning (SF), mental health (MH), bodily pain (BP), and vitality (VT). A score of 50 was judged to be equivalent to the general population while scores above or below this threshold indicated that a corresponding difference from the normal population was present. Using norm-based scores, we compared the HRQOL of patients with a sample from a general population. Patients were also asked to assess how they currently perceive their personal future health outlook as one of three options: positive, negative, or unchanged. The available medical documentation and survey data were used to analyze the disease course and evaluate its activity by the Birmingham Vasculitis Activity Score (BVAS) score (BVAS v.3; range, 0-56 patients. available at http://www.vasculitis.org/disease.htm) [14]. Patients with BVAS $<2$ were assumed to be in disease remission and included to the study. The survey packages were mailed to personal home addresses with return postage paid. All subjects gave informed consent to participate prior to inclusion in the study. Patients answered the survey and mailed it back to the hospital. The study protocol complied with the Helsinki Declaration and was approved by the Jagiellonian University Ethics Committee.

Statistical analysis was performed using Statistica (Statsoft, v10.0) software. Descriptive statistical analyses were performed for all. Ninety-five percent confidence intervals were also calculated on the basis of the Poisson distribution. The distributions of the response variables were checked for normality and transformations were performed as appropriate. When the pattern of the results observed using the transformed scores was similar to the results obtained from the raw data analysis, the raw data analysis is presented; if different, we report the results obtained using the transformed scores. Nonparametric tests were used as appropriate where the normality assumptions could not be 
met. The data were analyzed using Mann-Whitney $U$ tests and Fisher's test. $p$ values less than 0.05 were considered as statistically significant.

\section{Results}

A hospital database search identified 30 patients diagnosed with EGPA between July 1998 and December 2011. Each case was confirmed according to the ACR criteria for diagnosis of eosinophilic granulomatosis with polyangiitis and all of these patients were treated by a single medical team throughout the duration of their disease. From among the 30 patients who received survey packages, 26 replied and one was reported to be deceased. All of the respondents were included in this study. There were 26 patients ( 9 males and 17 females) with a mean age of $46 \pm 13$ years. The mean disease duration was $63 \pm 42$ months. All patients were confirmed to be currently in complete remission with a score of $<2$ on the BVAS. The baseline characteristics of the study population are show in Table 1. Additionally, a case-by-case review of medical records for confirmation of ACR criteria revealed asthma in 26 patients (100\%), sinusitis in 22 patients $(85 \%)$, eosinophilia in peripheral blood in 26 patients $(100 \%)$, mono or poly-neuropathy in $16(62 \%)$ and pulmonary eosinophilic infiltration in $20(77 \%)$. A tissue biopsy was performed in 13 cases (50\%); all results confirmed presence of vasculitis. The employment history of the study population is presented in Table 2 . The majority of patients were employed at diagnosis $(n=18,69 \%)$, but at time of the study only $50 \%(n=13)$ were employed ( $p=\mathrm{NS})$. Among those working at diagnosis, one working patient $(n=$ $1,100 \%$ ) changed jobs during the course of their disease, and did so because of EGPA. Among the unemployed, all three patients who were unemployed before diagnosis found

Table 1 Baseline characteristics of study population

\begin{tabular}{lc}
\hline Total patients & $n=26$ \\
Females, $n(\%)$ & $17(65 \%)$ \\
Males, $n(\%)$ & $9(35 \%)$ \\
Median age at diagnosis (years \pm SD, range) & $41 \pm 14(15-67)$ \\
Females & $39 \pm 10(19-60)$ \\
Males & $45 \pm 19(15-67)$ \\
Median age at study (years \pm SD, range) & $46 \pm 13(18-69)$ \\
Females & $44 \pm 10(22-62)$ \\
Males & $51 \pm 16(18-69)$ \\
Median duration of disease at survey & $63 \pm 42(6-180)$ \\
$\quad$ (months \pm SD, range) & \\
Females & $57 \pm 32(6-120)$ \\
Males & $75 \pm 57(12-180)$
\end{tabular}

Presented as absolute numbers and percentage of total. Age presented in years $\pm \mathrm{SD}$ with range new jobs despite developing EGPA and were working at the time of study. However, two of them were not able to work in their field due to EGPA and one of them was not affected by this diagnosis when returning to work (data not shown). Additionally, seven patients who previously worked became pensioners, and all seven (100\%) of them did so because of the impact of EGPA on their capacity to work. One working patient at time of diagnosis retired but this was not due to EGPA. The overall clinical picture of the study population throughout the course of their disease is presented in Table 3. The majority of subjects complained of sinus $(85 \%)$ and dyspnea $(85 \%)$ at diagnosis but were bothered mainly by sinus ( $54 \%$ ) and neuropathy (35\%) symptoms at the time of study.

Overall, the results of the SF-36 survey pictured by the tracings in Fig. 1, revealed patients with EGPA had lower quality of life parameters across all eight dimensions when compared with a normal population. Unemployment was associated with lower HRQOL (Fig. 1b). Only the VT dimension had a score that was almost identical to the normal population ( 48.8 vs. 50 ). Next, Table 4 shows the SF-36 survey results after patients were grouped into three separate categories according to their perceived personal future health outlook (available options: positive, unchanged, and negative). This breakdown revealed reverse trends by the two major component scores (MCS and PCS) of the SF-36 instrument. The MCS showed a correlation with a positive health outlook highlighted by an increase from 38.4 (below the population norm) in patients with a negative outlook to 50.4 (equal to population norm) in patients with a positive outlook. In contrast, the PCS decrease showed a negative trend with scores falling from 45.5 (below the population norm) in patients with negative future outlook to 41 (below the population norm) in patients with a positive outlook. The PF score decrease from 51.3 (equal to population norm) in patients with a negative outlook to 41 (below the population norm) in patients with positive outlook was found to be the only statistically significant difference $(p=0.01)$.

The breakdown of patients according to perceived future health outlook is presented in Table 5. Overall, there were 11 patients who had a positive outlook, 9 thought it would remain unchanged and 6 had a negative outlook. Global HRQOL was lower in patients with a negative outlook (Fig. 1b). There were no significant differences between males ( $n=4,44 \%$ positive) and females ( $n=7,41 \%$ positive). Their current work situation also did not seem to influence their outlook with employed (46\% positive) and unemployed ( $38 \%$ positive) having similar opinions regarding their future disease course. Two characteristics associated with a positive health outlook were young age ( $<50$ years) and shorter disease course. Approximately $36 \%$ of older patients ( $>50$ years) had positive outlook compared to $47 \%$ of younger patients 
Table 2 Employment profile of study population at diagnosis and time of study

\begin{tabular}{|c|c|c|c|c|c|}
\hline \multirow{5}{*}{ Employed } & \multicolumn{2}{|c|}{ At time of diagnosis } & \multicolumn{2}{|c|}{ At study time } & \multirow[t]{2}{*}{ Changes due to EGPA } \\
\hline & $\begin{array}{l}\text { Median } \\
\text { age }\end{array}$ & $41 \pm 10(26-57)$ & Median age & $42 \pm 8(34-57)$ & \\
\hline & Male & 13 & Male & 9 & \\
\hline & Females & 5 & Females & 4 & \\
\hline & Total & 18 & Total & 13 & $\begin{array}{l}\text { Patients who changed jobs because } \\
\text { of EGPA: } 2(100 \%) n(\% \text { of total } \\
\text { patients who changed jobs })\end{array}$ \\
\hline \multirow[t]{4}{*}{ Unemployed } & $\begin{array}{l}\text { Median } \\
\text { age }\end{array}$ & $34 \pm 9(23-41)$ & Median age & $\mathrm{n} / \mathrm{a}$ & \\
\hline & Male & 2 & Male & 0 & \\
\hline & Females & 1 & Females & 0 & \\
\hline & Total & 3 & Total & 0 & $\begin{array}{l}\text { Patients who lost their jobs because } \\
\text { of EGPA: } 0(0 \%) n(\% \text { of total } \\
\text { new unemployed })\end{array}$ \\
\hline Retired & 3 & & 4 & & $\begin{array}{l}\text { Newly retired because of EGPA: } 1 \\
(100 \%) n \text { (\% of newly retired) }\end{array}$ \\
\hline Pensioners & 0 & & 7 & & $\begin{array}{r}\text { New pensioner because of EGPA: } 7 \\
(100 \%) n(\% \text { of new pensioners })\end{array}$ \\
\hline Students & 2 & & 2 & & $\begin{array}{l}\text { New students: } 0(0 \%) \\
n(\% \text { of new students })\end{array}$ \\
\hline
\end{tabular}

$(<50$ years). The most significant differentiating factor was the length of duration of disease where patients with a longer course of disease ( $30 \%$ positive) were much less likely to have a positive outlook than those with shorter ones $(50 \%$ positive). These correlations were, however, not statistically significant.

\section{Discussion}

The aim of this study was to evaluate the current quality of life in EGPA patients and how this factor impacts their future health outlook. The patients overall quality of life was lower than the normal population across all dimensions of the SF-36 survey. This is the first study to directly investigate HRQOL in eosinophilic granulomatosis with polyangiitis patients via a standardized method (SF-36) that yields results which allow for direct comparison with other related diseases. Although EGPA patients have been included as part of larger studies [8], they made up an insignificant proportion of total study subjects and results may not accurately reflect the quality of life of these patients. When compared to other vasculitic diseases, these results show similar patterns to previously reported studies of diseases such as GPA [9] which revealed a similarly decreased level quality of life, most noticeably in patients who were
Table 3 Symptoms history and characteristics of study population

Included were self-reported symptoms reported on custom questionnaire. Results include absolute number of patients and percentage of total $(n=26)$. Empty fields denote that no patients reporting corresponding symptom. Patients were able to name more than one symptom in first and second column but only one in third column

\begin{tabular}{lccc}
\hline & $\begin{array}{l}\text { Symptoms at } \\
\text { diagnosis }\end{array}$ & $\begin{array}{l}\text { Dominant symptoms } \\
\text { (over last } 12 \text { months) }\end{array}$ & $\begin{array}{l}\text { Worst symptoms since } \\
\text { diagnosis (one symptom) }\end{array}$ \\
\hline Cough, $n(\%)$ & $20(77 \%)$ & $5(19 \%)$ & $3(11.5 \%)$ \\
Dyspnea, $n(\%)$ & $22(85 \%)$ & $7(27 \%)$ & $11(42 \%)$ \\
Fever, $n(\%)$ & $18(69 \%)$ & 0 & - \\
Malaise, $n(\%)$ & $25(96 \%)$ & $1(4 \%)$ & $3(11.5 \%)$ \\
Post-nasal drip, $n(\%)$ & $4(15 \%)$ & $4(15 \%)$ & - \\
Sinusitis, $n(\%)$ & $22(85 \%)$ & $14(54 \%)$ & - \\
Hay fever, $n(\%)$ & $22(85 \%)$ & $3(12 \%)$ & - \\
Paranasal sinus abnormality, $n(\%)$ & $8(31 \%)$ & $2(8 \%)$ & - \\
Neuropathy, $n(\%)$ & $16(62 \%)$ & $9(35 \%)$ & - \\
Weight loss, $n(\%)$ & $12(46 \%)$ & 0 & $2(8 \%)$ \\
Skin changes, $n(\%)$ & $11(42 \%)$ & 0 & $1(4 \%)$ \\
Pain, $n(\%)$ & $10(38 \%)$ & $5(19 \%)$ & - \\
Sight/hearing/smell disorders, $n(\%)$ & $1(4 \%)$ & $2(8 \%)$ & - \\
\hline
\end{tabular}


Fig. 1 Spydergrams of SF-36 results of EGPA patients compared to the general population grouped according to perceived health outlook (a) and employment status (b). PCS physical component summary, $M C S$ mental component summary, $G H$ general health, $P F$ physical functioning, $R P$ role physical, $B P$ bodily pain, $V T$ vitality, $S F$ social functioning, $R E$ role emotional, $M H$ mental health
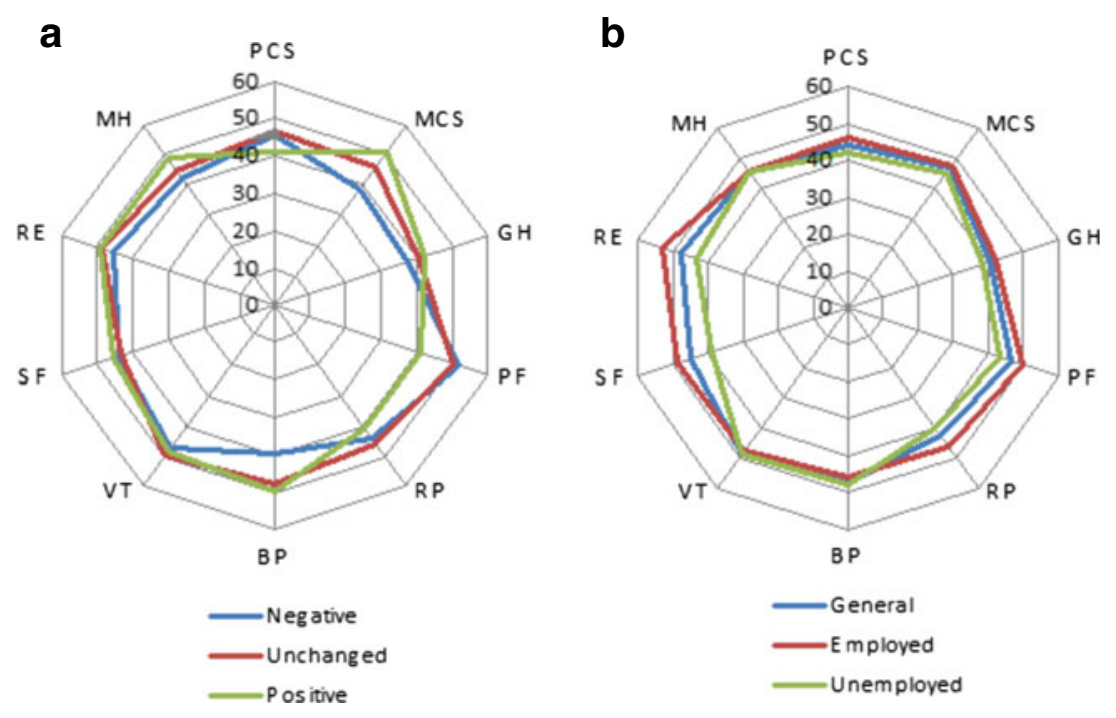

PCS - physical component summary; MCS - mental component summary; GH - general health; $\mathrm{PF}$ - phisicial functioning; RP -role physical; BP - bodily pain; VT - vitality; SF - social functioning; RE - role emotional; $\mathrm{MH}$ - mental health. unemployed $[15,16]$. A large study of ANCA-associated vasculitides found significantly decreased HRQOL at diagnosis most notably linked with neurologic involvement [8]. Finally, a Japanese study confirmed that even though quality of life improved over the 18 months the patients were included in a clinical trial, the SF-36 results were still below the population norm [17]. Taken together, the level of HRQOL decrease across the various vasculitic diseases, including the results of the present study, is similar in nature and in magnitude.

A major complaint among EGPA patients are symptoms of dyspnea and cough which are typically asthma related. In comparison, a large $(n=586)$ and very recent analysis of the Epidemiological Study on the Genetics and Environment of
Asthma cohort via SF-36 analysis revealed that HRQOL in well-controlled asthma patients remains relatively unaffected by their condition, where patients with uncontrolled asthma are the only ones who have a significant difference in HRQOL at time of study when compared to patients with well-controlled asthma [18]. These results are similar to those obtained in this study which shows that asthma symptoms do not significantly influence the HRQOL. In fact, we performed a multivariable analysis which revealed that the only significant determinant of HRQOL is peripheral nervous system involvement, confirming previous results described by Walsh et al. [8]. Other affected organs systems like the cardiovascular system, respiratory system, sinuses, kidneys, as well as long-term immunosuppression did not

Table 4 Indexed scores of the SF-36 survey's eight dimensions grouped according to patient's future health outlook

Future health outlook

\begin{tabular}{|c|c|c|c|c|c|c|}
\hline & Negative $(n=6)$ & $p$ & Unchanged $(n=9)$ & $p$ & Positive $(n=11)$ & $p$ \\
\hline MCS (mental component score) & $38.4 \pm 14.4$ & 0.17 & $45.6 \pm 9.2$ & 0.64 & $50.4 \pm 7.4$ & 0.12 \\
\hline PCS (physical component score) & $45.5 \pm 5.6$ & 0.73 & $46.5 \pm 8.9$ & 0.59 & $41.0 \pm 10.4$ & 0.4 \\
\hline GH (general health) & $37.3 \pm 7.8$ & 0.39 & $40.5 \pm 10.6$ & 0.97 & $41.7 \pm 7.3$ & 0.55 \\
\hline PF (physical functioning) & $51.3 \pm 5$ & 0.23 & $49.7 \pm 8.7$ & 0.10 & $41.0 \pm 8.7$ & 0.01 \\
\hline RP (role physical) & $43.1 \pm 8.5$ & 0.97 & $45.2 \pm 11$ & 0.38 & $40.4 \pm 8.5$ & 0.38 \\
\hline BP (bodily pain) & $39.4 \pm 9.4$ & 0.29 & $47.3 \pm 13$ & 0.87 & $49.3 \pm 12.3$ & 0.92 \\
\hline VT (vitality) & $46.7 \pm 13.7$ & 0.9 & $49.3 \pm 9.3$ & 0.81 & $47.6 \pm 9.2$ & 0.71 \\
\hline SF (social functioning) & $43.6 \pm 17.1$ & 0.9 & $42.9 \pm 10.2$ & 0.61 & $45.1 \pm 13.8$ & 0.71 \\
\hline RE (role emotional) & $44.9 \pm 14.9$ & 0.75 & $48.4 \pm 13.5$ & 0.68 & $48.8 \pm 7.9$ & 0.89 \\
\hline MH (mental health) & $41.7 \pm 12.5$ & 0.44 & $44.5 \pm 9$ & 0.53 & $48.5 \pm 6.7$ & 0.2 \\
\hline
\end{tabular}

Data expressed as mean value $\pm \mathrm{SD}$ 
Table 5 Characteristics of patient subgroups categorized according to differences in perception of future health outlook

Future health outlook

\begin{tabular}{llll}
\hline & $\begin{array}{l}\text { Negative } \\
(n=6)\end{array}$ & $\begin{array}{l}\text { Unchanged } \\
(n=9)\end{array}$ & $\begin{array}{l}\text { Positive } \\
(n=11)\end{array}$ \\
\hline $\begin{array}{lll}\text { Gender } \\
\quad \text { Male }(n=9)\end{array}$ & $2(22 \%)$ & $3(33 \%)$ & $4(44 \%)$ \\
$\quad \begin{array}{l}\text { Female }(n=17) \\
\text { Age }(\text { current })\end{array}$ & $4(24 \%)$ & $6(35 \%)$ & $7(41 \%)$ \\
$<50$ years $(n=15)$ & $2(13 \%)$ & $6(40 \%)$ & $7(47 \%)$ \\
$>50$ years $(n=11)$ & $4(36 \%)$ & $3(27 \%)$ & $4(36 \%)$ \\
Duration of disease & & & $8(37.5 \%)$ \\
$<66$ months $(n=16)$ & $2(12.5 \%)$ & $6(30 \%)$ & $3(30 \%)$ \\
$>66$ months $(n=10)$ & $4(40 \%)$ & & $6(46 \%)$ \\
Employment & & $5(38 \%)$ & $5(38 \%)$ \\
Employed $(n=13)$ & $2(15 \%)$ & $4(31 \%)$ & \\
Unemployed $(n=13)$ & $4(31 \%)$ &
\end{tabular}

Results presented as absolute number of patients and percentage of each category $n(\%)$

impact the HRQOL at time of study. Although asthma and its symptoms were not associated with a significant influence on the HRQOL at time of study in our analysis, these symptoms were mentioned as the most bothersome throughout the entire course of the disease (Table 3). Previous work by our group describing the course of asthma in patients with EGPA revealed an atypical course of this disease [11]. This study found that although EGPA patients suffer from severe asthma at the moment of diagnosis; these symptoms gradually subside over time and remain well controlled.

Also, employment status has been confirmed as an important determinant of HRQOL in past studies. Interestingly, in our study, employment did not influence either the current HRQOL or the future health outlook in a statistically significant manner. Other findings include a large proportion of the study population (7/26) requiring to apply for a pension. This did not translate into a statistically significant difference in HRQOL measures.

Notably, a novel contribution of this study is the examination of patients' perceived future health outlook as an independent factor resulting from current HRQOL. The results obtained in this study suggest that the MCS is the most reliable predictor in reference to patients' perception of their own health. Patients with higher MCS scores felt more optimistic about the future while patients with low PCS were likely not to be more pessimistic about their future health. Thus, it seems fair to conclude that MCS carries substantially more weight in shaping patients' perceived future health outlook. In other words, patients who feel most positively about their future health outlook, currently exhibit the highest quality of life from a psychological point of view. This result is surprising and should serve to alert physicians that, paradoxically, patients with a positive frame of mind may actually be in a worse physical health state than their counterparts who seem more pessimistic about their future health. The finding that patients with the lowest PCS scores remained positive about their future outlook may be explained by the fact that, at diagnosis, the disease manifested itself in its most severe form. Hence, these patients have witnessed a significant improvement over the years and remain hopeful of further improvements to come. In contrast, patients who may have had lighter forms of the disease and whose quality of life remained initially unaffected could account for the fact that these patients have a bleak outlook going forward. Arguably, these conclusions could only be considered accurate once a statistically significant correlation is achieved by working with a larger sample group. Limitations of this analysis are mainly due to the small number of patients included in this study ( $n=$ 26) as EGPA is a very rare disease. This makes the statistical correlation analysis difficult. Additionally, a baseline SF-36 result obtained at the time of diagnosis could have been useful to identify expectation bias. Establishing this baseline may distinguish if patients who currently suffer from the lowest physical well-being yet perceive their future health positively do so because they are the ones which have improved the most over the years. Their improvements could skew their future outlook as they may be looking forward to similar improvements in the future. In the same vein, a follow-up questionnaire at 1 year would likely confirm certain trends and extract new trends which have gone unnoticed within the limited amount of data gathered. Finally, an obvious amelioration of the validity of these results would be an expansion of the sample size to include more patients and gain increased statistical power.

\section{Conclusion}

Patients with EGPA had decreased HRQOL across all eight dimensions of the SF-36. Patients with higher MCS felt more positive about their future health, while patients with low PCS were likely not to feel negatively about their future health. Despite being in disease remission, patients with EGPA had decreased quality of life, which in turn influenced their perception of their future health outlook. A larger study population with would be required to confirm these findings in a statistically significant manner.

Acknowledgments We would like to thank the Rand Corporation (www.rand.org) for their complimentary contribution of the SF-36 questionnaire used in this study.

Disclosures None. 
Open Access This article is distributed under the terms of the Creative Commons Attribution License which permits any use, distribution, and reproduction in any medium, provided the original author(s) and the source are credited.

\section{References}

1. Szczeklik W, Jakieła B, Adamek D, Musial J. (2011) Cutting edge issues in the Churg-Strauss syndrome. Clin Rev Allergy Immunol

2. Szczeklik W, Miszalski-Jamka T, Mastalerz L et al (2011) Multimodality assessment of cardiac involvement in Churg-Strauss syndrome patients in clinical remission. Circ J 75(3):649-655

3. Szczeklik W, Sokołowska B, Mastalerz L et al (2010) Pulmonary findings in Churg-Strauss syndrome in chest X-rays and high resolution computed tomography at the time of initial diagnosis. Clin Rheumatology 29(10):1127-1134, Epub 2010 Jul 12

4. Lanham JG, Elkon KB, Pusey CD et al (1984) Systemic vasculitis with asthma and eosinophilia: a clinical approach to the ChurgStrauss syndrome. Medicine (Baltimore) 63(2):65-81

5. Guillevin L, Pagnoux C, Seror R et al (2011) The five-factor score revisited: assessment of prognoses of systemic necrotizing vasculitides based on the French vasculitis study group (FVSG) cohort. Medicine (Baltimore) 90(1):19-27

6. World Health Organization (2006) Basic Documents, Forty-fifth edition, Supplement. Constitution of the World Health Organization. WHO, Geneva

7. Brazier JE, Harper R, Jones NM et al (1992) Validating the SF-36 health survey questionnaire: new outcome measure for primary care. BMJ 305(6846):160-164

8. Walsh M, Mukhtyar C, Mahr A et al (2011) Health realated quality of life in patients with newly diagnosed antineutrophil cytoplasmic antibodyassociated vasculitis. Arthritis Care Res (Hoboken) 63(7):1055-1061
9. Tomasson G, Boers M, Walsh M et al (2012) Assessment of healthrelated quality of life as an outcome measure in granulomatosis with polyangiitis (Wegener's). Arthritis Care Res (Hoboken) 64(2):273-279

10. Yazdany J, Yelin E (2010) Health-related quality of life and employment among persons with systemic lupus erythematosus. Rheum Dis Clin North Am 36(1):15-32

11. Szczeklik W, Sokołowska BM, Zuk J et al (2011) The course of asthma in Churg-Strauss syndrome. J Asthma 48(2):183-187

12. Stoll T, Sutcliffe N, Klaghofer R et al (2000) Do present damage and health perception in patients with systemic lupus erythematosus predict extent of future damage? A prospective study. Ann Rheum Dis 59:832-835

13. Masi AT, Hunder GG, Lie JT et al (1990) The american college of rheumatology 1990 criteria for the classification of Churg-Strauss syndrome (allergic granulomatosis and angiitis). Arthritis Rheum 33:1094-1100

14. Mukhtyar C, Lee R, Brown D et al (2009) Modification and validation of the Birmingham vasculitis. Activity score (version 3). Ann Rheum Dis 68(12):1827-1832

15. Faurschou M, Sigaard L, Bjorner JB et al (2010) Impaired health related quality of life in patients treated for Wegener's granulomatosis. J Rheumatol 37(10):2081-2085

16. Reinhold-Keller E, Herlyn K, Wagner-Bastmeyer R et al (2002) Effect of Wegener's granulomatosis on work disability, need for medical care, and quality of life inpatients younger than 40 years at diagnosis. Arthritis Rheum 47(3):320-325

17. Suka M, Hayashi T, Kobayashi S et al (2012) Improvement in health-related quality of life in MPO-ANCA-associated vasculitis patients treated with cyclophosphamide plus prednisolone: an analysis of 18 months of follow-up data from the JMAAV study. Mod Rheumatol 22(6):877-884

18. Siroux V, Boudier A, Bousquet J et al (2012) Asthma control assessed in the EGEA epidemiological survey and health-related quality of life. Respir Med 106(6):820-828 